Pearce, T. W. \& Powell, E. O. (1951). J. gen. Microbiol. 5, 91-103.

\title{
New Techniques for the Study of Growing Micro-organisms
}

\author{
By T. W. PEARCE and E. O. POWELL \\ Microbiological Research Department, Ministry of Supply, Porton, Wiltshire
}

\begin{abstract}
SUMMARY: A method is described for the examination of growing micro-organisms by vertical and oblique incident illumination using an illuminator of the 'Universal Illuminator' (Messrs Cooke, Troughton and Simms) type. A moist chamber has been developed to enable this method to be used for the routine examination of bacterial cultures, and examples are given of both aerobic and anaerobic cultures on the surface of solid nutrient media, preferably containing nigrosine.

This method has also been used for following spore germination of Bacillus subtilis in a liquid medium. A method using Cellophane as a support for growth is also described. Certain organisms can be grown on Cellophane 'windows' made in the caps of Bijou bottles containing a liquid medium; this apparatus is suitable for the repeated but not continuous examination of young growths.
\end{abstract}

The methods commonly used for the continuous observation of growing micro-organisms under the microscope suffer from the defects of difficulty or inconvenience in manipulation, and often of unnatural restraint upon the organisms themselves. In this paper we describe the application of two systems of illumination which have long been current among metallurgists for observations on opaque specimens, viz. 'vertical' and 'oblique incident' illumination. Even in biological work these are far from novel. Thus the 'Lieberkühn' is a simple form of oblique illuminator; it is 200 years old, and was originally conceived by Descartes (Carpenter, 1891). While pleas for their more extended use have appeared from time to time (e.g. Vonwiller, 1927) they have not in our opinion been applied to the extent that they deserve. Our object therefore is to suggest that the efficient and convenient modern form of apparatus might well become a routine tool in microbiological work, not largely confined, as seems to have been so far, to occasional highly specialized investigations.

The optical equipment is described briefly, partly because of its unfamiliarity to some workers, partly because its practical form will be seen to conduce greatly to the ease of manipulation which can be attained. Apart from a description of general features, we consider in detail a number of applications; examples are chosen principally to indicate the range that can be covered though each has some point of intrinsic interest.

\section{Vertical and oblique incident illumination}

In vertical illumination, light from an illuminated iris, brought nearly parallel by means of a lens, is directed towards a thin glass plate lying on the axis of the microscope immediately behind the objective. The plate is tilted so as to reflect part of the light directly downwards through the objective, which then forms an image of the iris on the surface of the specimen under observation. 
If the specimen reflects specularly, only a brightly illuminated circle is seen; irregularities of contour appear dark wherever the surface departs sufficiently from normality to the axis, or scatters some of the incident light.

Oblique incident illumination is the same as ordinary dark ground, except that the hollow cone of light is applied from above instead of below the specimen. A parallel hollow cylindrical beam is formed by an annular diaphragm in front of the lamp, and reflected by an annular mirror in the axis of the microscope; the down-going beam surrounds the light path from the objective to the eye. It is focused by a bispherical condenser as in the usual dark-ground system. The objective is specially constructed in a narrow tube passing through a central hole in the condenser and supported by a spider. A more detailed description can be found in, for instance, Langeron (1948).

The image formed by the one method of illumination is, roughly speaking, the negative in the photographic sense of that formed by the other. In both cases 'metallurgical' objectives-computed for use without a cover-glass-are used.

Vertical illumination is normally capable of giving information only about the surface of a specimen; the change of refractive index on passing from air to specimen is large, and so most of the light reaching the eyepiece is that which has been reflected at the surface. Optical inhomogeneities within a transparent object are seen with difficulty, if at all; they usually reflect less light than an air-solid interface, and that little is lost in the much brighter reflexion from the surface.

Oblique illumination is capable of reaching objects embedded in transparent solid or liquid media, since the ground remains dark. The useful depth is limited by the deterioration in image quality that is tolerable. With a $4 \mathrm{~mm}$. objective, about $50 \mu$. is the maximum.

We have throughout used a microscope designed for metallurgical work, i.e. one with a stage which can be raised and lowered by rack and pinion, which will accommodate large objects, and whose whole top surface can be moved in both directions at right angles to the microscope axis. Further, although the illuminators can be fitted with self-contained lamps, an external and more powerful source is better; once this has been aligned with the mirror, the coarse adjustment of the body cannot afterwards be moved, and stage focusing is a necessity. Most of our work has been carried out with the 'Universal Illuminator' (Messrs Cooke, Troughton and Simms) which embodies both optical systems in one fitting. A common mirror is used, its centre being clear and its outer part silvered. Two tubes carrying the necessary stops and lens are mounted side by side on a slide attached to the body; by interposing one or the other between lamp and mirror either a central or an annular beam is admitted. Thus either method of illumination can at once be brought into play by a simple manual adjustment. The objective need not be changed, and the darkground condenser remains in position.

This arrangement has two great advantages. First, the rapid interchange of vertical and oblique illumination makes it possible to obtain without refocusing and searching all the information available from a given object under these conditions. Secondly, focusing itself is much facilitated when only dark-ground 
observations are required; it is carried out as follows. The vertical illuminator is brought into position and its field iris nearly closed. The specimen is then raised towards the objective until the image of the iris is seen sharply defined on its surface. This occurs suddenly and cannot easily be missed. On then switching over to dark ground it is certain that the surface is still in focus though the particular field may be optically empty. There is thus no danger of damage to the front lens of the objective, or of contamination from live pathogenic organisms in the specimen.

The apertures of the objectives used with oblique incident illumination are limited by the fact that part of the solid angle above the specimen must be occupied by the illuminating beam. The highest dry power available is a $4 \mathrm{~mm}$., N.A. 0.65, initial magnification 45. With vertical illumination there is no such inherent limitation; there is provision in the 'Universal Illuminator' for replacing the dark-ground condenser and special objectives by objectives of normal construction, which may be of the highest power and aperture.

The special virtue of these forms of illumination is thus that they facilitate direct examination of micro-organisms growing on, or at a short distance below, the surfaces of liquid or solid media. There is little restriction on the form-especially the depth-of the container. For following the growth of a micro-organism which is left in position under the microscope, we prefer to work in a room held at a constant temperature of $35^{\circ}$. The physiological discomfort is less irksome than the restriction on speed and accuracy of manipulation imposed by a 'hot box', and the absence of temperature gradients confers many advantages. A heat filter between lamp and specimen is essential in work with growing organisms; without it they are rapidly killed. A poor filter allows fogging of the objective by vapour rising from the medium. We have found a $6 \mathrm{~mm}$. total thickness of Chance's ON 19 heat-absorbing glass to be entirely satisfactory.

\section{Examination of growth on solid media}

Media for oblique incident illumination. When the oblique incident illuminator is used on the surface of agar, the image seen suffers from lack of contrast owing to Tyndall scattering in the gel, which produces a fairly bright field. Further, ordinary media are never free from foreign bodies, crystals, or globules of fat; these introduce confusing and irrelevant detail. The difficulty can be overcome by adding to the medium a dye which absorbs light strongly (cf. Klieneberger \& Smiles, 1942); for this purpose we have used nigrosine. A $10 \%$ $(\mathrm{w} / \mathrm{v})$ stock solution of nigrosine in water is Seitz-filtered after adjustment of the $\mathrm{pH}$ to neutrality. One volume of the solution is added to 10-20 vol. of molten medium. Though it is convenient to treat fairly large amounts of routine media in this way, we use a more economical method with special media of which perhaps only one or two plates are required occasionally. A small quantity of a common medium such as tryptic meat agar, with nigrosine, is melted, and a thin layer poured over the surface of the special medium; the plate is then allowed to stand for $24 \mathrm{hr}$. or more, when sufficient diffusion will have taken place for the uppermost surface to have acquired the particular properties of 
the underlying medium. Plates made from media prepared in this way are extremely opaque; foreign bodies and other inclusions give little trouble since those at a depth of more than a few microns are not illuminated. It is important, however, that the surface should be as free from blemish as possible. Usually it is sufficient to pour the medium at $60^{\circ}$ or greater; this allows a considerable time for settling before it solidifies. The common impurities in the surface itself, notably collections of fat globules, become familiar by experience, and for many purposes can be disregarded. Where there is likely to be confusion, we pass the whole medium while hot through a Seitz filter before pouring, thus obtaining plates whose surfaces are almost entirely featureless over large areas.

Tests have been made of the ability of organisms to grow on media containing nigrosine. The test species ranged in general robustness from Bacillus subtilis to Neisseria meningitidis, and in complexity of food requirements from Azotobacter chroöcoccum to Haemophilus pertussis. Plates of untreated medium and of the medium with added nigrosine were inoculated in pairs and incubated. The results are shown in Table 1. With only three organisms was the growth obviously restricted namely, Brucella suis, Br. tularensis, and Pfeifferella malle $i$-and in these only where the inoculum was sparse. One other result deserves notice. The growth of some organisms is inhibited by small quantities of gentian violet. Our Staphylococcus aureus failed to grow on a medium containing $0.001 \%$ of the dye, but with added nigrosine growth proceeded normally. Nigrosine combines with triphenylmethane dyes (Knaysi, 1944), and it seems that it thereby neutralizes their bacteriostatic properties.

Since nigrosine is usually available in bacteriological laboratories, and because it proved to be so widely applicable as a light absorbent, we made no extended tests of other materials. It should not be difficult to find substitutes in cases where its presence is objectionable.

Media for vertical illumination. With vertical illumination, no special treatment of the bulk medium is called for, since only the surface itself can be seen. Foreign particles and corrugations in the surface must of course be avoided. Organisms which grow in or upon the surface of a medium are best examined with vertical illumination; in oblique light they produce very intense scattering and flare, and details are obscured.

\section{Observations of growth on solid media}

It is a very simple matter to watch the growth of a young colony of microorganisms on a nigrosine-nutrient agar plate in the conditions described above at a magnification of 400-600 diameters. For the best results, however, attention must be paid to the size of the inoculum and to the dryness of the medium. Evaporation from the latter causes recession of the surface (which must be avoided during photographic exposures), a hardening of the surface layers through concentration of the agar, and a consequent progressive change in the appearance of bacterial growths. Some form of moist chamber is therefore desirable. Our apparatus is described in the following account of our general procedure, using Streptococcus lactis as an example. 


\section{Table 1. Groroth of organisms on media containing nigrosine}

\begin{tabular}{|c|c|c|c|c|}
\hline \multirow[b]{2}{*}{ Organism } & \multirow[b]{2}{*}{ Medium } & \multicolumn{3}{|c|}{ Growth } \\
\hline & & $\begin{array}{l}\text { Without } \\
\text { nigrosine }\end{array}$ & $\begin{array}{c}\text { With } \\
\text { nigrosine } \\
\text { incorporated }\end{array}$ & $\begin{array}{l}\text { With } \\
\text { nigrosine } \\
\text { layer }\end{array}$ \\
\hline $\begin{array}{l}\text { A. chroöcoccum (freshly isolated } \\
\text { from soil) }\end{array}$ & $\underset{\text { TMA }}{\text { PMM }}$ & $\begin{array}{l}+ \\
+\end{array}$ & $\begin{array}{l}+ \\
+\end{array}$ & $\dot{.}$ \\
\hline $\begin{array}{l}B \text {. anthracis (4 fully virulent and } \\
1 \text { avirulent strain) }\end{array}$ & $\begin{array}{l}\text { GCY } \\
\text { TMA }\end{array}$ & $\stackrel{+}{+}$ & + & $\dot{.}$ \\
\hline B. licheniformis & $\begin{array}{l}\text { TMA } \\
\text { GL } \\
\text { PA }\end{array}$ & $\begin{array}{l}+ \\
+ \\
+\end{array}$ & $\begin{array}{l}+ \\
+ \\
+\end{array}$ & $\dot{.}$ \\
\hline $\begin{array}{l}\text { Bacillus group } 9 \text { species other than } \\
\text { anthracis and licheniformis }\end{array}$ & $\begin{array}{l}\text { TMA } \\
\text { PA }\end{array}$ & $\begin{array}{l}+ \\
+\end{array}$ & + & $\dot{.}$ \\
\hline Bact. coli & $\begin{array}{l}\text { TMA } \\
\text { MacConkey's } \\
\text { Desoxycholate }\end{array}$ & $\stackrel{+}{+}$ & $\dot{t}$ & $\stackrel{\dot{+}}{(+)}$ \\
\hline Br. suis (a fully virulent strain) & $\begin{array}{l}\text { TMA } \\
\text { Liver extract agar }\end{array}$ & $\begin{array}{l}+ \\
+\end{array}$ & $(+)$ & $(\dot{+})$ \\
\hline Br. tularensis (a fully virulent strain) & $\begin{array}{l}\text { TMA } \\
\text { Francis's* }\end{array}$ & $\begin{array}{l}(+) \\
+\end{array}$ & $(+)$ & $(\dot{+})$ \\
\hline Chr. prodigiosum & TMA & + & + & . \\
\hline Cl. botulinum & $\begin{array}{l}\text { TMA } \\
\text { PSB }\end{array}$ & $\begin{array}{l}+ \\
+\end{array}$ & + & $\dot{+}$ \\
\hline Cl. welchii & $\begin{array}{l}\text { TMA } \\
\text { PSB }\end{array}$ & $\begin{array}{l}+ \\
+\end{array}$ & + & $\dot{+}$ \\
\hline C. diphtheriae (gravis) & $\begin{array}{l}\text { TMA } \\
\text { Hoyle's }\end{array}$ & + & + & $\dot{+}$ \\
\hline C. xerosis & Loeffler's & + & + & . \\
\hline H. pertussis & Blood agar & + & • & + \\
\hline Leuconostoc sp. & TMA & + & + & - \\
\hline $\begin{array}{l}\text { M. tuberculosis (laboratory strains of } \\
\text { human, bovine and avian, and several } \\
\text { freshly isolated from sputa) }\end{array}$ & $\begin{array}{l}\text { Löwenstein-Jensen's } \\
\text { Dorset egg }\end{array}$ & + & : & $\begin{array}{l}+ \\
+\end{array}$ \\
\hline M. phlei & TMA & + & + & - \\
\hline$N$. gonorrhoeae & Chocolate agar & + & . & + \\
\hline N. meningitidis & Chocolate agar & + & - & + \\
\hline $\begin{array}{l}P \text {. pestis (a fully virulent and an } \\
\text { avirulent strain) }\end{array}$ & $\begin{array}{l}\text { TMA } \\
\text { PSB }\end{array}$ & $\stackrel{+}{+}$ & + & $\dot{+}$ \\
\hline Pf. mallei & TMA & + & $(+)$ & . \\
\hline Pr. hydrophilus & TMA & + & + & . \\
\hline Ps. pyocyanea & $\begin{array}{l}\text { TMA } \\
\text { Loeffler's }\end{array}$ & $\begin{array}{l}+ \\
+\end{array}$ & + & . \\
\hline S. typhi & $\begin{array}{l}\text { TMA } \\
\text { MacConkey's } \\
\text { Desoxycholate }\end{array}$ & $\begin{array}{l}+ \\
+ \\
+\end{array}$ & + & $\dot{+}+$ \\
\hline Staph. aureus & $\begin{array}{l}\text { TMA } \\
\text { TMA }+0.001 \% \\
\text { gentian violet }\end{array}$ & \pm & + & $\dot{\cdot}$ \\
\hline Strep. faecalis & TMA & + & + & • \\
\hline Strep. lactis & TMA & + & + & . \\
\hline Strep. pyogenes ( $\beta$-haemolytic type) & TMA & + & + & - \\
\hline
\end{tabular}

Abbreviations. $\mathrm{PMM}=\mathrm{a}$ medium containing only mannitol, potassium phosphates, a trace of molybdate and well-washed agar. (Topley \& Wilson, 1946). TMA = tryptic meat agar. CCY = Gladstone \& Fildes, (1940) tryptic casein yeast medium. $\mathrm{GL}=\mathrm{a}$ chemically-defined medium containing only glucose, citrate and lactate as sources of carbon. (Hills, Belton \& Blatchley, 1949). PA=peptone agar. PSB=TMA with addition of glucose and peptic sheep blood.

* For Francis's medium see Stitt, Clough \& Clough (1938).

In some cases direct microscopical examination showed that the early growth was not adversely affected by nigrosine. These are indicated above by printing the designation of the medium in heavy type. 
A plate of tryptic meat agar containing 0.5-1\% of nigrosine is poured and allowed to set. Without further drying it is inoculated at its centre with a small loopful of a suspension of Strep. lactis containing $c .10^{5}$ organisms $/ \mathrm{ml}$. The plate is left with its lid ajar until evaporation and absorption have left no free liquid at the site of inoculation. Then an aluminium ring, being a $5 \mathrm{~mm}$. length cut from a tube $5-7 \mathrm{~cm}$. in diameter, is heated in a flame and dropped concentrically on the surface of the plate. The agar melts locally and seals the

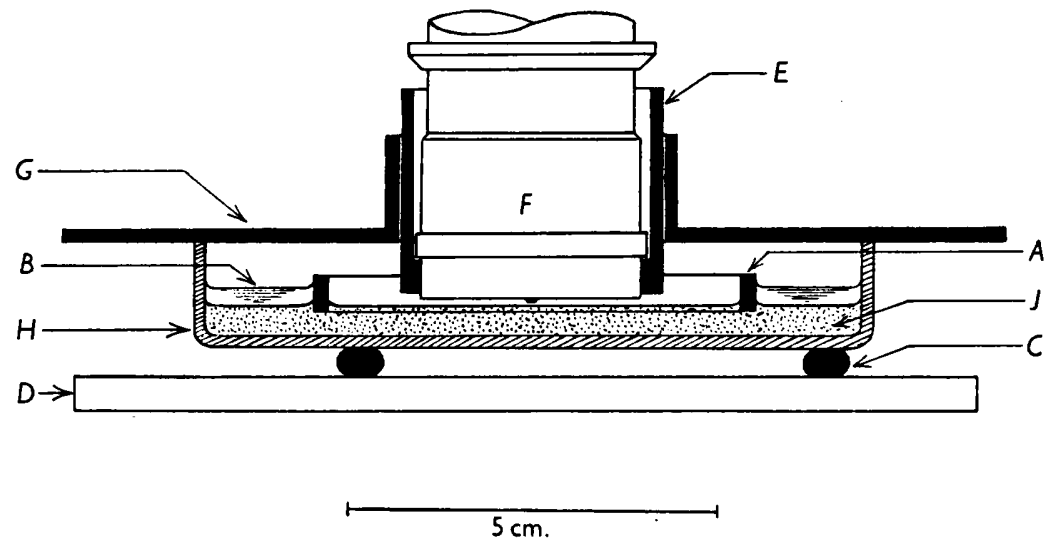

Fig. 1. Section of moist chamber. $A$, aluminium ring; $B$, water ; $C$, plasticine balls ; $D$, microscope stage; $E$, sleeve fixed to $F$, body of illuminator (enclosing objective); $G$, loose cover sliding on $E$ and $H$, Petri dish; $J$, medium.

ring to the surface. The annular space outside the dam thus formed is partly filled with water, and the plate is placed on three balls of plasticine on the stage of the microscope. A cylindrical brass sleeve ( $E$, Fig. 1$)$, is fixed by set screws to the body of the dark ground condenser $(F)$, which is applied to the surface of the agar through a loose cover $(G)$. The dish and cover are then free to move vertically for focusing; the dish can also slide transversely beneath the cover which remains fixed meanwhile. The plasticine $(C)$ allows the dish to be levelled so that the plane of its edge is normal to the axis of the microscope. There is very little loss of vapour from the chamber so formed; in practice the inoculated area of the medium remains steadily in focus for long periods, the slight excess vapour pressure of the water outside the dam over that of the medium itself counteracting any tendency to evaporation from the central area.

With oblique incident illumination and the highest available power of the microscope the individual cells of Strep. lactis can be seen to grow into chains lying for the most part just beneath the surface of the medium. The organisms are well separated and cell division is easily observed, but no finer details. When the chains contain more than a very few organisms, they begin to buckle as the individuals divide; the mechanical resistance to motion becomes too great to permit perfect longitudinal extension and ultimately each chain forms a very complex pattern. But none ever appears to branch; up to the highest degree of convolution that the eye can follow, a single cell of the inoculum 
produces a colony consisting of a single chain. Thus the plane of symmetry implied in the mode of cell division of the streptococcus is maintained approximately in its direction through many generations. Pl. 1, fig. 1, shows a typical young colony formed after $3 \mathrm{hr}$. incubation at $35^{\circ}$.

When conditions are a little drier, growth takes place on the surface of the medium instead of below it, and is best seen by vertical illumination. Pl. 1, fig. 2 , shows a young colony growing on a plate dried by storage at $0^{\circ}$ for a few days, and inoculated by allowing the organisms to sediment from a cloud produced by spraying, so that no free water was added with the inoculum. Otherwise the conditions were the same as in the previous example. The chain of cells forms the backbone of a ridge whose flanks are composed of the polysaccharide elaborated in quantity by this species.

The post-fission movements of rod-shaped organisms, as described and classified by Graham-Smith (1910) for example, are very easily followed by this method, and the whole architecture of a colony can be traced out. While the method might thus be of some diagnostic value, the variations within any one species may be great, and will depend on the chemical and physical environment and also on purely local conditions determined by the size of the colony already formed.

The growth of Bacillus subtilis is interesting in this connexion. When first deposited on a plate, the ellipsoidal spore resembles a diplococcus by oblique illumination, the scattered light apparently radiating from two points near its poles (Pl. 1, fig. 3). When after $1 \frac{1}{2}-2 \mathrm{hr}$. the vegetative cell begins to grow out, its image is much less bright. The two halves of the spore case do not usually separate completely, though they may do so under pressure from the growing organisms. In any case they retain the high refractivity of the original spore indefinitely (Pl. 1, fig. 4). The subsequent unhampered growth is usually in long chains which form loops and cusps under the longitudinal pressure of growth. At this stage they are scarcely distinguishable from the corresponding forms of $B$. anthracis. As the population density increases, the cohesion of the chains is insufficient to maintain their form; they break apart and the sections slide over each other to produce a colony which consists for the most part of closely packed sheets of organisms. In contrast, B. anthracis chains are much stronger and retain their integrity over long distances in spite of considerable mechanical constraint from neighbours (Pl. 1, figs. 5,6). When the B. subtilis colony contains a few hundred organisms, say after $\mathbf{4 - 5} \mathrm{hr}$. incubation, motility becomes evident. The close-packed areas are surrounded by a moat of free liquid, a few microns wide and deep, which is presumably extracted from the agar osmotically by the products of metabolism; in it detached organisms swim freely. A few static chains grow out but usually recurve to join the colony again, enclosing loculi which, if small, are filled with a thin layer of liquid. The loculi become populated by motile organisms which arrive from beneath the adjacent sheet of cells; each such organism, after a few minutes violent excursion within the loculus, ultimately rests in contact with the confining wall (either by adhesion or more likely by failure of the motor apparatus) and in this way an extension of the close-packed colony is produced. The whole of this process of piecemeal 
extension has an aspect of purposeful organization. As the colony grows further it occurs less and less often, and ultimately the edge is without outgrowths, even though the central mass may be in violent swirling motion. Nevertheless, this high degree of organization is largely a product of the environment. With a more dilute agar the organisms can grow to an appreciable depth, and the regularity of packing is lost, whereas if the surface is harder or drier, or both, the organisms lie upon it, and the mechanical resistance to motion principally determines the growth pattern. On a chemically-defined medium containing only citrate, lactate and glucose as sources of carbon (Hills, Belton \& Blatchley, 1949), B. subtilis of the same strain does not form welldefined chains; the cells are short and soon separate after fission, producing a pattern suggestive of brickwork (Pl. 1, fig. 7). The same pattern is sometimes found on ordinary media, and growth may change from one to the other during the development of a colony.

\section{Use of the Conway Unit for bacterial cultures}

The Conway Unit (Conway \& Byrne, 1933) is a circular glass dish (diameter c. $6.5 \mathrm{~cm}$.; internal depth $c .0 \cdot 8 \mathrm{~cm}$.) with a raised annulus in its floor, originally intended for microchemical analytical work. It is more economical of media, simpler to use, and more generally applicable when used as a culture plate than the Petri dish and aluminium ring. The central cup (diameter $c .3 .5 \mathrm{~cm}$; internal depth $c .0 \cdot 6 \mathrm{~cm}$.) is filled almost to overflowing with nutrient medium ( $5 \mathrm{ml}$.) so as to present a convex surface; any liquid or solid if required may be placed in the outer annulus. The greater part of the surface of the medium is accessible to the oblique incident illuminator. As an example of the use of the Conway Unit we take the remarkable ability of $B$. subtilis to grow on the surface of a medium quite free from liquid and of great mechanical rigidity.

The centre of a Conway Unit was filled with tryptic meat medium containing $6 \%$ agar. The outer annulus was filled with a mush of solid sodium chloride and water. This mixture maintains a humidity of $75 \%$ over itself. The covered unit was allowed to stand for $3 \mathrm{hr}$. at $35^{\circ}$ and then inoculated with $B$. subtilis spores by exposure to the cloud from a sprayed suspension in a small closed chamber. At this stage a good deal of water had been extracted by the salt from the medium; the sedimented spores evidently stood high on the surface (Pl. 2, fig. 8) which was quite brittle as shown by its cracking under pressure from a glass rod. Although water vapour immediately above such a surface must of course be in equilibrium with it, and therefore not much below saturation, the high rate of evaporation will cause a considerable increase in the concentration of solutes at the surface. The spores were thus in contact with an unusually concentrated medium, but over a small part only of their area. Yet many of them germinated after the normal period and subsequently the reproduction time in the logarithmic phase was about $30 \mathrm{~min}$. as against $20 \mathrm{~min}$. in more ordinary circumstances. The hindrance to motion across the surface was evinced by the production of distorted cells (Pl. 2, fig. 8) and by the throwing up at an early stage of a second layer of cells overlying the first (Pl. 2, fig. 9). 


\section{Observations on anaerobes}

Anaerobic conditions are not difficult to establish in the closed chamber on the microscope stage. For this purpose we use a circular metal dish to contain the culture vessel (Fig. 2); the objective and condenser are applied through the same loose but close-fitting cover as in Fig. 1. The wall of the dish is pierced

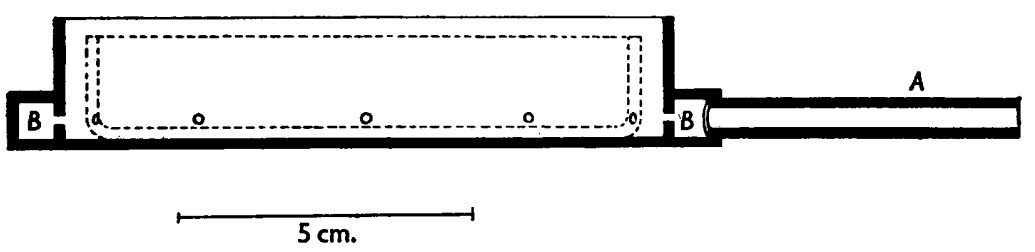

Fig. 2. Section of dish for anaerobic culture under the microscope. $A$, side tube for admission of nitrogen, etc.; $B, B$, annular space for distribution of gas.

with a ring of holes giving upon an annular channel $(B, B, F i g .2)$ through which gas can be passed into the interior. The chamber is fed with a slow stream of nitrogen which has been passed through an oxygen absorbent (e.g. titanous chloride). It may also when necessary be saturated with water vapour, or contain an addition of carbon dioxide. The arrangement suffices for the growth of the more exacting anaerobes but the lag phase is likely to be protracted unless steps are taken to remove dissolved oxygen from the medium before inoculation.

Pl. 2, fig. 10, shows the edge of a colony of Clostridium welchii grown on a nigrosine tryptic meat agar plate in an atmosphere of nitrogen saturated with water. As with Bacillus anthracis, the chains rarely break, but the distinguishing feature here is the intertwining of adjacent chains, which do not restrict themselves so rigidly to one plane.

\section{Observation of growth of spores at liquid surfaces}

In connexion with the problem of the enhancement of the virulence of B. anthracis by detergents (Barnes, 1947), we carried out a few experiments on the growth of spores at liquid surfaces, of which the following illustrates the complementary use of vertical and oblique incident illumination. Spores of $B$. subtilis were dispersed as an aerosol by spraying a clean water suspension. The cloud was allowed to settle for a few minutes on to the surface of tryptic meat broth contained in a well on a microscope slide. When the surface was examined under oblique illumination, the spores were surrounded each by a strong flare; by vertical light they were easily seen to be floating with a considerable portion of their bulk standing out of the surface. They were therefore not much wetted. The experiment was repeated with an aerosol made by spraying a spore suspension containing $5 \%$ of 'Tergitol 4', a detergent in which the active compound is principally a branched chain alkyl sodium sulphate (Messrs General Metallurgical \& Chemical Corporation, London). Each spore in the cloud thus carried a small quantity of detergent. The sedimented spores in this case were hardly to be seen by vertical illumination, but by oblique 
illumination gave a well-defined 'diplococcus' image; they had been almost completely wetted on contact with the medium, though the majority remained attached to the surface. This difference in the area of contact was without significant effect on the subsequent development; in both cases vegetative cells appeared beneath the surface after $1 \frac{1}{2}-2 \mathrm{hr}$. incubation. Unfortunately we were unable to make satisfactory photographs of these phenomena, owing to disturbance of the liquid surface by vibration and convection currents.

\section{Cellophane as a support for growing organisms}

Thin permeable membranes have been used from time to time as a support for, or barrier against, growing micro-organisms in order to allow them to be manipulated independently of their culture medium, and vice versa. Thus Gladstone (1948) used Cellophane tubes to separate cultures of $\boldsymbol{B}$. anthracis from their nutrient medium, which was kept flowing over the surface of the Cellophane remote from the organisms, and thus remained sterile; and McKercher \& Reed (1948) grew organisms on sheets of Cellophane in order to avoid the use of agar and to enable them to be cleanly stripped from the medium afterwards.

For direct microscopical examination of growing organisms by vertical illumination, Cellophane has some advantages over ordinary media as a support. By comparison with agar it is moderately hard and is not much altered in this respect by changes in the humidity of the surrounding atmosphere. Organisms do not grow into the surface, and can be examined without disturbance under the highest dry powers of the microscope. There is, however, the disadvantage that the normal growth pattern of the colony is obliterated by surface tension forces which cause the organisms to stick together.

Culture on Cellophane under the microscope. We have used Cellophane as a support in two general methods of microscopical observation. One of thema culture chamber allowing of very complete control of the environment of the organism-will be described in a later communication. The other is intended for the repeated but not continuous examination of young growths, more especially when many specimens are being studied at the same time. It consists of a standard 'Bijou' ( $\frac{1}{4}$ oz.) bottle (Messrs Gallenkamp, London) with a Cellophane window in the screw cap. The bottle contains the growth medium and the outer surface of the window is inoculated with the organism under test. A disk of thin rubber is placed inside the screw cap on the thick washer supplied and a $1 \mathrm{~cm}$. hole is punched simultaneously through both layers of rubber and the cap itself, using a sharp-edged, deeply recessed punch against a sheet of fibre. The washers are removed, and the thinner one replaced first in the cap. This is followed by a disk of Cellophane, 20-25 $\mu$. thick, and then the thicker washer (Fig. 3). One or two ml. of medium are put in the bottle and the cap screwed on lightly; the whole is then autoclaved. The outer surface of the Cellophane may then be inoculated. The inoculum is allowed to evaporate nearly to dryness, the bottle inverted in a boiling tube or other narrow vessel, and incubated in this position. At first the Cellophane is wrinkled, but as the medium warms up and its vapour pressure increases, the window forms a taut 
dome on which it is easy to focus the microscope. When the bottle is removed for examination, the Cellophane is usually seen to be covered with small droplets of liquid; these evaporate rapidly when the bottle is in the upright position, and the organisms on the surface are very clearly seen under the microscope by vertical illumination. There is sufficient liquid underneath the Cellophane to prevent complete drying during a short examination, after which the bottle may be restored to the inverted position and growth allowed to proceed as before. Even if only a single observation has to be made, e.g. for presence or absence of growth, there is still a considerable advantage in the short time normally elapsing between inoculation and the formation of recognizable colonies, and in the rapidity with which a long series of cultures may be examined. In experiments lasting only a few hours, the usual sterile precautions are for most purposes superfluous; the chance of seeing a contaminant under the microscope is remote, and infection of the medium can in its early stages rarely affect the behaviour of the organisms being studied.

In this way we have been able to study the action of certain antibiotics. By comparison of serial dilu-

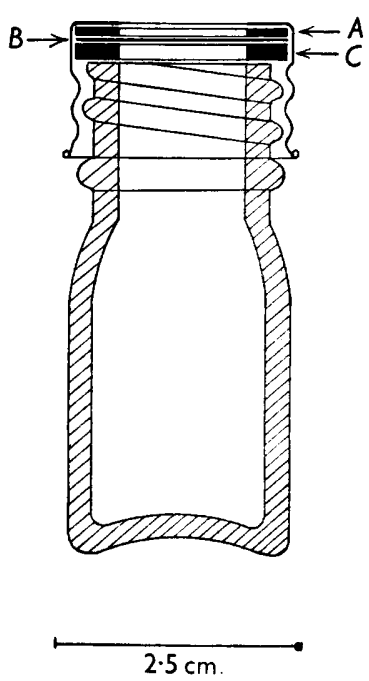

Fig. 3. Section of screw-cap bottle with Cellophane window. $A$, thin rubber washer; $B$, Cellophane disk; $C$, thick rubber washer. tions of standard and unknown preparations it is possible to make estimates of concentration within a few hours. The accuracy is rather greater than in an all-or-nothing growth test, since the degrees of aberration produced in the test organism near the critical concentration can be assessed roughly. Pl. 2, fig. 11, shows some forms of $B$. subtilis grown for $3 \mathrm{hr}$. on Cellophane over peptone water containing 0.003 units penicillin $/ \mathrm{ml}$. In one case the two halves of the spore residue have divided, and have remained attached one to each end of a short chain of cells. More remarkable is the presence of two very distorted growths, one undergoing lysis, in these circumstances providing evidence that the cells are quite flaccid. Under the actual conditions of growth, the organisms are immersed in droplets of liquid. When the bottles are returned to the upright position these droplets evaporate and surface tension tends to keep the organisms within their boundaries. The consequent bending of the organisms can be seen to occur at this stage. Only a moderate curvature, if any, is produced in healthy growths (compare Pl. 2, fig. 12).

Among the limitations on the use of Cellophane is its small pore size; Madras, Mason \& McIntosh (1949) estimated the average pore diameter to be about $2 \cdot 8 \mathrm{~m} \mu$. only. This is a severe restriction on the size of molecules which will pass through; penicillin passes easily, lysozyme very slowly, haematin not at all. But no other membranes that we have been able to test will replace Cellophane. Nylon and various cellulose esters are less readily wetted by water, 
and films of these substances will not permit the passage of medium unless there is liquid on both sides. Membranes from animal tissues and eggs are otherwise suitable, but have too coarse a structure to present a smooth background.

The impermeability of Cellophane to large molecules is demonstrated in our last example. Mechanically defibrinated horse, cow or human blood will not support growth of $\boldsymbol{B}$. subtilis. Spores deposited from the air on to the surface swell and break open, but then appear to become encysted, and grow no further. Up to at least $5 \mathrm{hr}$. after inoculation they remain viable, and when transferred to an ordinary medium will grow normally. Blood to which citrate or oxalate has been added will support growth, though rather poorly. If, however, the spores are separated by Cellophane from mechanically defibrinated blood, they germinate normally and subsequently grow very freely (PI. 2, fig. 13). It will be noted that short detached vegetative forms are produced (cf. Pl. 1, fig. 7). The inhibitor is thus of fairly large size; it may be produced in the clotting process or removed by anticoagulants.

We are indebted to Messrs British Cellophane Ltd., Bridgwater, Somerset, for a large sample of good quality Cellophane; to our instrument maker, Mr G. Clement; and to Miss Nora Harris, who took the photographs. Publication is by permission of the Chief Scientist, Ministry of Supply.

\section{REFERENCES}

BARNES, J. M. (1947). The development of anthrax following the administration of spores by inhalation. Brit. J. exp. Path. 28, 385.

Carpenter, W. B. (1891). The microscope and its revelations, 7th ed. by W. $\mathrm{H}$. Dallinger. London: J. and A. Churchill.

Conway, E. J. \& BYrNe, A. (1933). An absorption apparatus for the micro-determination of certain volatile substances. I. The micro-determination of ammonia. Biochem. J. 27, 419.

Gradstone, G. P. (1948). Immunity to anthrax. Production of the cell-free protective antigen in Cellophane sacs. Brit. J. exp. Path. 29, 379.

Gradstone, G. P. \& Fildes, P. (1940). A simple medium for general use without meat extract or peptone. Brit. J. exp. Path. 21, 161.

Graham-Smith, G. S. (1910). The division and post-fission movements of bacilli when grown on solid media. Parasitology, 3, 17.

Hills, G. M., Belton, F. C. \& Blatchley, Errol D. (1949). Ayfivin: production in chemically defined media and comparison with licheniformin. Brit. J. exp. Path. 30, 427 .

Kireneberger, E. \& Smiles, J. (1942). Some new observations on the developmental cycle of bovine pleuropneumonia and related microbes. J. Hyg., Camb., 42, 110.

Knaysi, G. (1944). Elements of Bacterial Cytology. Ithaca, N.Y., U.S.A.: Comstock Publishing Co.

Langeron, M. (1948). Précis de Microscopie, 7th ed. Paris: Masson et Cie.

Madras, S., Mason, S. G. \& McIntosh, R. L. (1949). A preliminary study of the permeability of Cellophane to liquids. Canad. J. Res. 27, (B), 764.

McKercher, D. G. \& REed, G. B. (1948). Surface growth of bacteria on Cellophane. Canad. J. Res. 26, (E), 330. 
Journal of General Microbiology, Vol. 5, No. 1
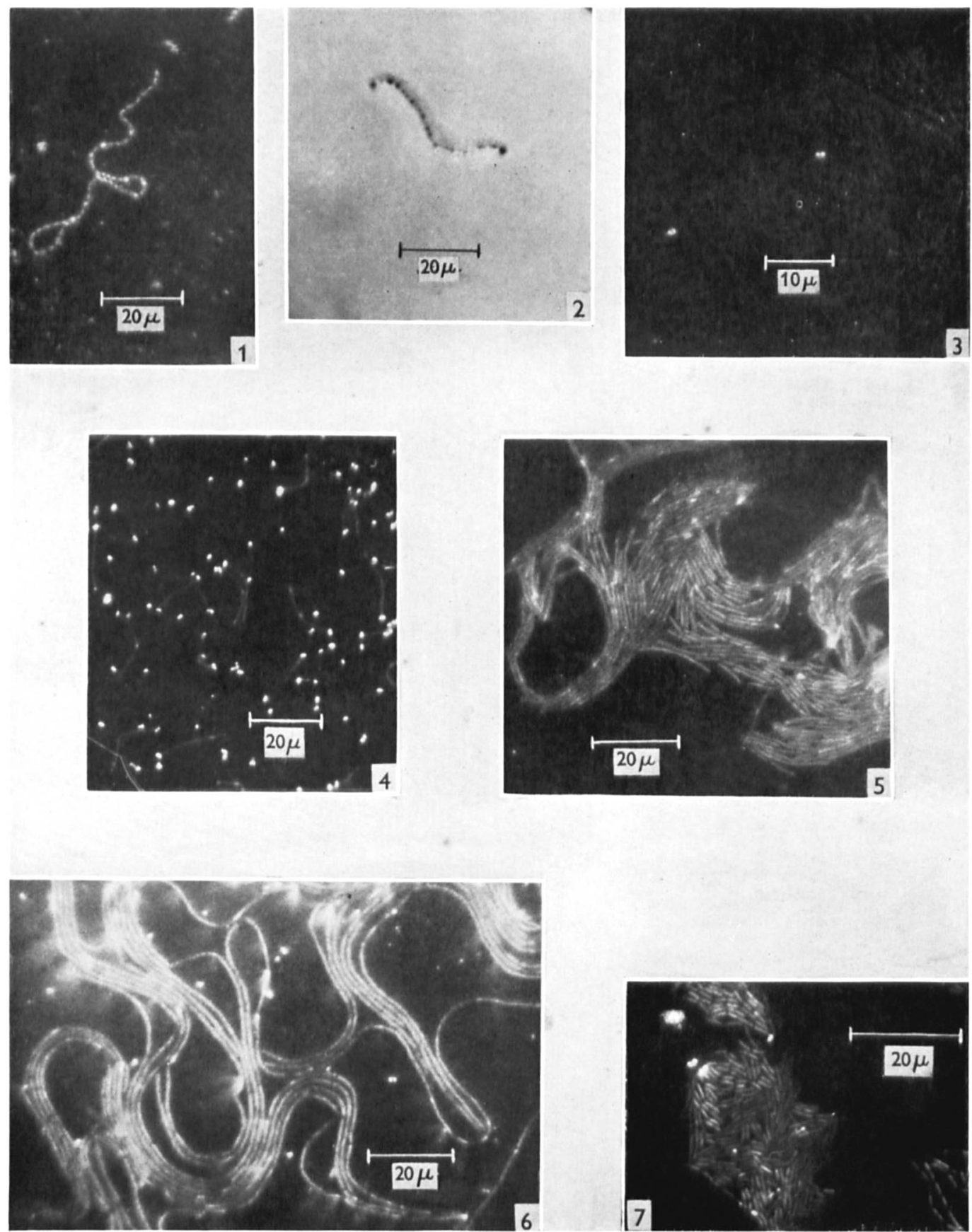

Figs. 1-7

T. W. Pearce and E. O. Powell-New techniques for studying growing bacteria. Plate 1 
Journal of General Microbiology, Vol. 5, No. 1
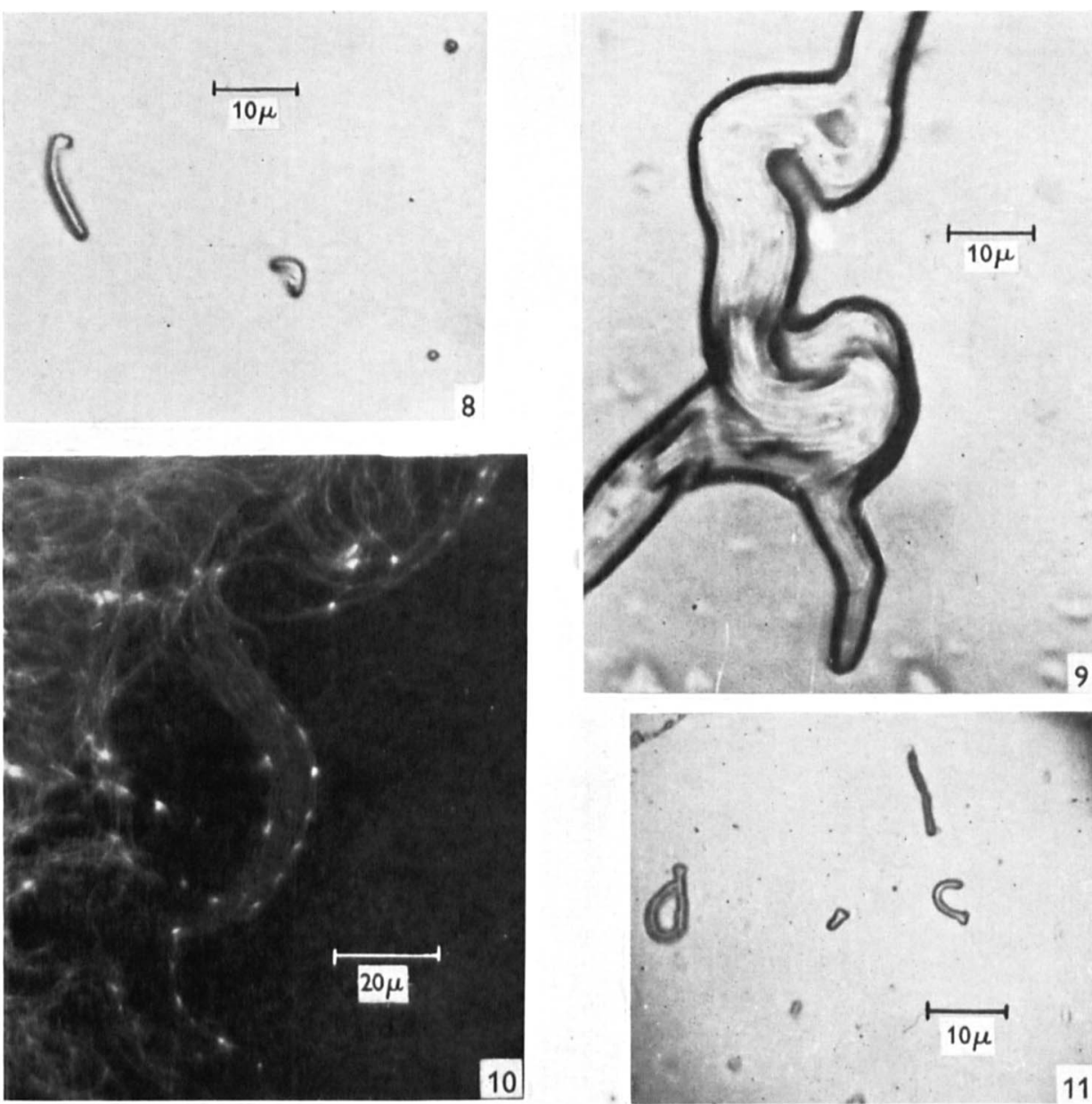

10
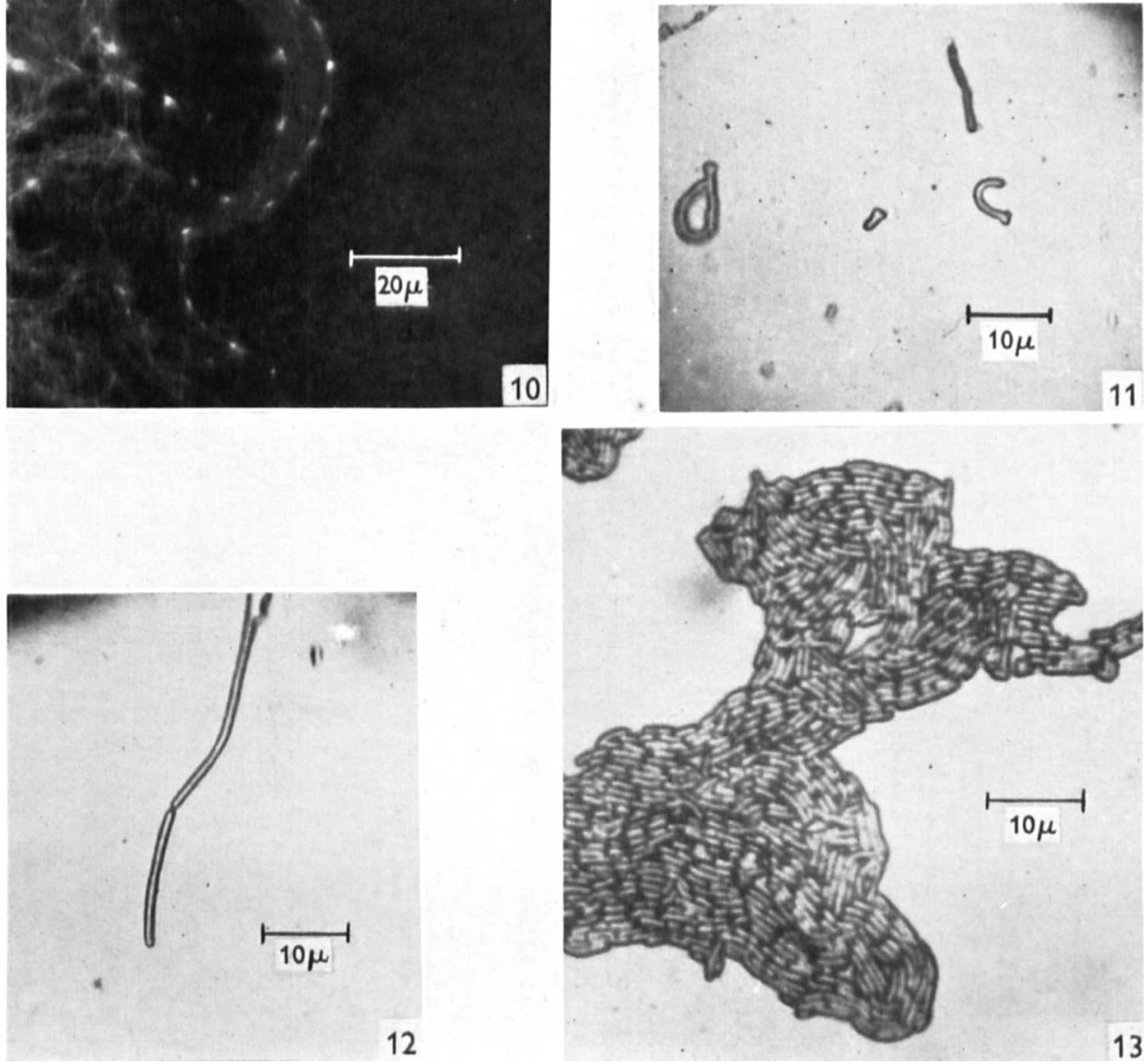

Figs, 8-13

T. W. Pearce and E. O. Powell-New techniques for studying growing bacteria. Plate 2 
Stitt, E. R., Clough, P. W. \& Clough, M. C. (1938). Practical Bacteriology, Haematology, and Animal Parasitology, 9th ed. Philadelphia, Pa., U.S.A.: The Blakiston Company.

Topley and Wilson's Principles of Bacteriology and Immunity (1946). 3rd. ed. Wilson, G. S. \& Miles, A. A. London: Edward Arnold and Co.

Vonwiller, P. (1927). Microscopy with incident light and its application to living objects. J. R. micr. Soc. (3), 47, 325.

\section{EXPLANATION OF PLATES}

\section{Pluate 1}

Fig. 1. Strep. lactis. $3 \mathrm{hr}$. at $35^{\circ}$ on nigrosine tryptic meat agar plate. Oblique incident illumination. Objective: Cooke, $\times 45,0.65$ N.A. achromat.

Fig. 2. Strep. lactis. As fig. 1 but on drier plate and with vertical illumination.

Fig. 3. B. subtilis. Spores on nigrosine medium, showing apparent diplococcal form. Optics as fig. 1.

Fig. 4. B. subtilis. Spores and young vegetative forms after $2 \mathrm{hr}$. at $35^{\circ}$ on nigrosine medium. Opties as fig. 1 .

Fig. 5. B. subtilis. Part of colony 6.5 hr. old on nigrosine peptone agar. Optics as fig. 1.

Fig. 6. B. anthracis. Part of colony $6.5 \mathrm{hr}$. old on nigrosine peptone agar. Optics as fig. 1 .

Fig. 7. B. subtilis. Colony $3 \mathrm{hr}$. old, grown on a chemically-defined medium. Short detached forms embedded in medium. Optics as fig. 1.

\section{Prate 2}

Fig. 8. B. subtilis. Spores and young growths on a very dry medium, showing a distorted cell and residues of spore-case. Vertical illumination. Objective: Beck, $\times 40,0.95$ N.A. apochromat.

Fig. 9. B. subtilis. Young colony on very dry agar medium, illustrating the piling up of cells at an early stage. Maximum contrast has been used in reproducing the photograph so as to bring out details within the boundary of the colony. Vertical illumination. Objective: Cooke, $\times 45,0 \cdot 65$ N.A. achromat.

Fig. 10. Cl. welchii. $16 \mathrm{hr}$. at $35^{\circ}$ on nigrosine tryptic meat agar plate. Edge of colony, characterized by twisting together of adjacent chains. Optics as fig. 1.

Fig. 11. B. subtilis. $3 \mathrm{hr}$. growth on Cellophane over peptone water containing 0.003 units penicillin/ml. Abnormal forms. Optics as fig. 9.

Fig. 12. B. subtilis. Outgrowth from a young colony grown on Cellophane over peptone water. Illustrating rigidity of healthy organism. Optics as fig. 8.

Fig. 13. B. subtilis. Colony of short detached forms grown on Cellophane over mechanically defibrinated human blood. Optics as fig. 9. 